\title{
An outbreak of gastroenteritis on board an oil drilling rig in Ghana
}

\author{
Michael N.K. Babayara ${ }^{1}$, Bright Addo ${ }^{2}$ \\ ${ }^{1} 37$ Military Hospital, Postgraduate College 37 Military Hospital, Accra, Ghana \\ ${ }^{2}$ University of Ghana School of Public Health, Accra, Ghana
}

\begin{abstract}
The article describes an outbreak of gastroenteritis which occurred on an oil drilling rig which operated in the Jubilee fields of Ghana (offshore Takoradi) between May 2011 and December 2013. An outbreak occurred on board approximately $10 \mathrm{~h}$ following a Christmas day buffet. Various Ghanaian and continental dishes were served during the occasion. Over a 3 day period, a total of 33 cases were recorded at the rig's sick bay. In order to describe the outbreak and determine its possible association with the meals served at the buffet, this case control study was conducted. A short questionnaire was used for data collection and a line list prepared for summarising data of cases. Samples of food could not be taken but water samples from multiple points of the rig's pipe water system were collected and cultured for coliforms. Data was analysed using Epi info version 7. Six foods appeared to be associated with the outbreak with odds ratios ranging from 1.2 to 2.7. The epidemic curve showed a propagated fashion of transmission and the outbreak was brought under control with public education on hand washing and disinfection of surfaces and door handles. The conclusion was that food items served at the buffet were not associated with the outbreak. Instead, a possible contamination of surfaces and door handles probably caused it. To prevent future occurrences, routine and regular hand washing and disinfection of surfaces and door handles is recommended.
\end{abstract}

(Int Marit Health 2018; 69, 1: 51-57

Key words: diarrhoeal outbreak, Sedco energy, oil rig, Ghana

\section{INTRODUCTION}

The outbreak of diarrhoeal diseases following festive occasions such as parties is very common, and offshore and marine units such as ships have not been spared a share of this phenomenon. Much as ships have long been recognised as sites for outbreaks of infectious diseases [1, 2], the same cannot be said about oil drilling rigs. However, the two share very common characteristics that make them equally prone to outbreak situations. Just like a cruise ship, a rig carries personnel from different places where infections could be acquired, brought on board and spread to other people. Also, their environments are such that spread of infections is easily facilitated; many people are living together in a confined space and eating together, sharing bedrooms and toilet facilities, dining facilities, drinking from one water supply, and perhaps inhaling expired air from each other. This places a large cohort of people at risk of a communicable disease at any particular point in time. These outbreaks are of particular importance because they could have very serious health consequences for crew members and result in heavy economic losses to companies.

For diarrhoeal disease outbreaks, studies conducted to identify the main factors underlying their outbreaks have implicated ineffective temperature control, infected food handlers, contaminated raw ingredients, cross contamination, and inadequate heat treatment [3]. Agents predominantly implicated also include bacteria (Salmonella spp., Shigella spp., E. coli, Vibrio spp., and Clostridium spp.), viruses, parasites and agents of unknown aetiology. Whereas these 
may be frequently implicated, not all outbreaks can be associated with a particular factor.

This article covers an outbreak of a diarrhoeal disease which occurred on an oil drilling rig which operated in the Jubilee fields of Ghana between May 2011 and December 2013. The installation has a maximum personnel on board (POB) capacity of 195 people. Being an industrial setting, people of varying professional backgrounds including engineers, safety specialists, administrators, welders, painters, drillers, environmental experts, caterers, etc. are usually found on board. These people come from various countries across the globe, and are adults of various age groups and race. It is a predominantly male environment, with females coming on board only occasionally. Periods of stay on board varies from person to person depending on the demands of their specific assignments but is generally not more than 35 continuous days.

Just like a cruise ship, most of the rig environment is enclosed with limited or no direct exposure to the outside environment. It has an accommodation section which houses offices, living areas, kitchen and dining areas, and cabins for sleeping. Personnel occupy cabins in pairs and sometimes in fours in the case of junior officers. The accommodation is compressed to prevent the influence of bad weather; and ventilation is through a central air condition system. A catering department is responsible for the provision of meals and all meals are only eaten at the dining hall, and not allowed outside this area. This kind of environment makes the rig an ideal setting for the rapid spread of contagious diseases. The Sedco energy itself has indeed seen minor outbreaks of various conditions including flu, diarrhoea, and chicken pox in the past.

The reported outbreak started almost $10 \mathrm{~h}$ following a buffet organised by the catering department to mark Christmas day on $25^{\text {th }}$ December, 2013. Cases of acute episodes of diarrhoea associated with vomiting, and abdominal cramps were first seen at the rig sick bay approximately $12 \mathrm{~h}$ following the buffet which was held from 17:00 $\mathrm{h}$ to 19:00 $\mathrm{h}$ the previous night.

Various Ghanaian and continental dishes were served during the buffet and included the following:

- starters: cream of tomato, basil parmesan soup with croutons, shrimp platter Marie-rose, king prawns and lobsters platters, mozzarella platters/salmon roll platters, assorted salads/suckling pig, and cold cuts of tenderloin of beef/cold turkey;

- main course: crown of roast stuffed turkey with pan roasted juice, grilled lobster and prawns with garlic butter slowly cooked a la plancha, grass cutter and snail green soup, creamed potatoes, sunshine potatoes, and stuffing and traditional accompaniments;

- desserts: assorted cheesecakes/Christmas cake, sweets, profiteroles pyramid, fresh fruit platter, and cheese and biscuits.
To understand the nature of the outbreak, and identify the main factors associated with it, this investigation was carried out. It was an unmatched case control study with controls taken randomly from personnel visiting the rig sick bay within the period that cases were reporting, but presenting with sicknesses other than gastroenteritis. The objective of this investigation was therefore to describe the gastroenteritis outbreak on the Sedco energy rig and to determine its possible association with the meals served at the buffet. It was hypothesized that there was an association between the buffet and the gastroenteritis outbreak on the rig.

\section{MATERIALS AND METHODS}

\section{DATA COLLECTION}

A short questionnaire was used for data collection and a line list prepared for summarising data of cases. Data captured included demographic details, race, and nationality, signs/symptoms, and onset and resolution dates, as well as foods eaten at the buffet.

Once someone presented and met the classification criteria, he was immediately attended to after which the investigation was explained to him and upon his consent, the questionnaire was administered. Samples of food could not be taken for laboratory analysis due to constraints with facilities in the offshore environment. However, a mandatory rapid diagnostic test for malaria was done for all cases presenting with fever.

Water samples from multiple points of the rig's pipe water system were collected and cultured for coliforms. Samples of Voltic mineral water (the main source of drinking water) were also analysed for coliforms. For ease of data capture and analysis, food items served were assigned codes (Table 1).

\section{SELECTION OF CASES AND CONTROLS}

For the purpose of this investigation, a case of diarrhoea was defined as an episode of three or more stools looser than normal in a $24 \mathrm{~h}$ period with/without vomiting, abdominal cramps, or fever. Cases were those who presented to the sick bay with symptoms meeting the case definition and who had partaken in the buffet. Controls were patients who presented to the sick bay during the period of the outbreak but with conditions other than gastroenteritis and who had partaken in the buffet.

\section{STATISTICAL ANALYSIS}

Data from questionnaires was entered into Epi info version 7 , reviewed for completeness and accuracy and analysed. Frequencies, proportions, and bivariate analysis were carried out at 95\% confidence interval. The line list was transported into excel and an epidemic curve was drawn. 
Table 1. Food items and their assigned codes

\begin{tabular}{ll}
\hline Food item & Code \\
\hline Cream of tomato, basil parmesan soup with croutons & ST1 \\
Shrimp platter Marie-rose & ST2 \\
King prawns and lobsters platters & ST3 \\
Mozzarella platters/salmon roll platters & ST4 \\
Assorted salads/suckling pig & ST5 \\
Cold cuts of tenderloin of beef/cold turkey & ST6 \\
Crown of roast stuffed turkey with pan roasted juice & MC1 \\
Grilled lobster and prawns with garlic butter & MC2 \\
slowly cooked a la plancha & \\
Grass cutter and snail green soup & MC3 \\
Creamed potatoes, sunshine potatoes, stuffing & MC4 \\
and traditional accompaniments & \\
Assorted cheesecakes/Christmas cake & DS1 \\
Sweets & DS2 \\
Profiteroles pyramid & DS3 \\
Fresh fruit platter & DS4 \\
Cheese and biscuits & DS5
\end{tabular}

Table 2. Distribution of cases $(n=33)$ by age, sex and race

\begin{tabular}{lll}
\hline Characteristics & Frequency & Percentage \\
\hline Age group [years]: & & \\
$20-30$ & 9 & $27.3 \%$ \\
$31-40$ & 14 & $42.4 \%$ \\
$41-50$ & 10 & $30.3 \%$ \\
Sex: & & \\
Male & 33 & $100 \%$ \\
Female & 0 & $0 \%$ \\
Race: & & \\
African & 9 & $27.3 \%$ \\
Caucasian & 18 & $54.5 \%$ \\
Other & 6 & $18.2 \%$
\end{tabular}

Table 3. Distribution of symptoms among cases

\begin{tabular}{lll}
\hline Symptom & Frequency & Percentage \\
\hline Diarrhoea & 100 & $100 \%$ \\
Vomiting & 11 & $33.3 \%$ \\
Abdominal cramps & 15 & $45.5 \%$ \\
Fever & 7 & $21.2 \%$ \\
Dehydration & 0 & $0 \%$
\end{tabular}

\section{ANALYSIS OF SAMPLES OF WATER}

Water samples were collected from taps in rooms of some cases and controls, the kitchen, the coffee room, the storage tanks, and the water making machine for analysis. Bottles of Voltic mineral water were also analysed. Aseptic techniques were used to avoid contaminating water samples. The analysis of water included incubating samples using $\mathrm{HACH}$ presence/absence broth ampoules for $48 \mathrm{~h}$ at $35^{\circ} \mathrm{C}$. This is an easy to use test for screening drinking water for total coliforms. Food samples were not available to be sampled for analysis. Excess food was processed and fed to fishes before the next morning when the outbreak started.

\section{ENVIRONMENTAL ANALYSIS}

A thorough inspection of the kitchen, the dining hall, the freezers and food processors, the cold stores and chillers in which raw foods are kept was done to establish if any protocols regarding food handling and hygiene standards were breached. Records of cleaning schedules, temperature monitoring of freezers and chillers, weekly hygiene inspections, and food container inspection records were reviewed thoroughly for possible clues. Rig engineers also conducted a thorough inspection of the sewage system for possible leakages.

\section{CONTROLLING THE OUTBREAK}

While investigation of the outbreak was going on; cases were being treated and followed up. An aggressive education campaign using power point presentations was carried out on the need for strict adherence to basic hygiene principles. Disinfection of door handles, stair case rails, etc. was carried out and portable hand sanitizers were distributed to all crew.

\section{RESULTS}

\section{BACKGROUND CHARACTERISTICS OF RESPONDENTS}

The outbreak lasted 3 days and the average duration of illness was $16 \mathrm{~h}$ with treatment. Attack rate was $17.4 \%$. Males had a $100 \%$ distribution because there were no females on board the installation at the time of occurrence. The age group most commonly affected was the 31 to 40 year old age group (Table 2), but the specific ages affected ranged from 20 to 54 years. The median age was 35 years and the modal was 32 years.

The installation generally has a predominant Caucasian population of approximately $90 \%$ (from POB records). This reflected in the distribution of the outbreak as more Caucasians were affected than any other race (Table 2).

\section{DIARRHOEA INCIDENCE}

Diarrhoea was the main symptom used in the case definition and therefore had an expected $100 \%$ distribution. However abdominal pain in this outbreak was a common 


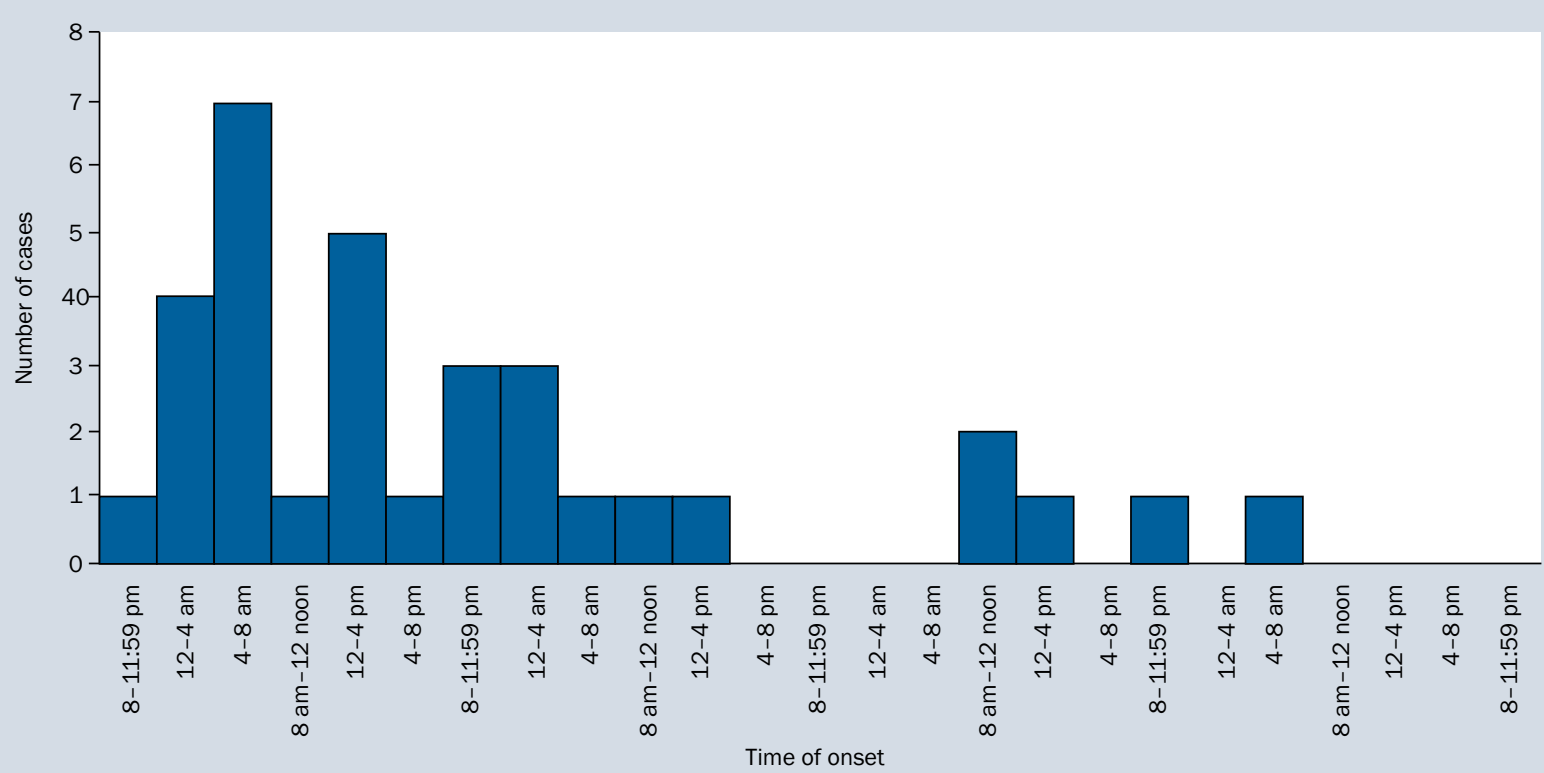

Figure 1. Epidemic curve showing cases per unit time

symptom having occurred in $45.5 \%$ of cases. Vomiting and fever were also significant symptoms but fortunately; there were no cases of dehydration as will normally be expected (Table 3). The most cases were recorded between 4 am and 8 am on the $26^{\text {th }}$ of December ( 7 cases). A graph plotted for number of cases per time unit generally shows an epi-curve with four different peaks; each peak lasting approximately $24 \mathrm{~h}$. The general trend is characterised by a rapid rise in cases over a $24 \mathrm{~h}$ period (from $8 \mathrm{pm}$ on 25/12/2013 to $4 \mathrm{pm}$ on $26 / 12 / 2013)$. This rapidly declined to zero as no further cases were reported between the afternoon of the $27^{\text {th }}$ and the dawn hours of the $28^{\text {th }}$ December. Few cases were seen following the $28^{\text {th }}$ December but generally the number of cases declined until the $29^{\text {th }}$ December when no further cases were reported and the outbreak thus ended (Fig. 1).

\section{FOODS SERVED AT BUFFET AND DIARRHOEA INCIDENCE}

In total, 14 different food items were served on the night (Table 4). These included various assorted continental and local Ghanaian cuisines. There were starters, main dishes and desserts. The most preferred starters, main course and dessert were cream of tomato, basil parmesan soup with croutons, grilled lobster and prawns with garlic butter slowly cooked a la plancha, and assorted cheesecakes/ /Christmas cake, respectively. Seventeen people ate the cream of tomato, basil parmesan soup with croutons and about 59\% of them became ill. Twenty nine people took grilled lobster and prawns with garlic butter slowly cooked a la plancha and $59 \%$ of these were ill. For those who preferred assorted cheesecakes/Christmas cake as a dessert, about $69 \%$ fell ill.

Of the different types of foods eaten, 6 appeared to be associated with the outbreak with odds ratios ranging from 1.2 to 2.7 (Table 4). The associated confidence intervals however had wide margins depicting great variability in the data. All confidence intervals also included 1 in the range of values, and all p-values were greater than 0.05 hence conferring lack of significance in the observed associations. Among the starters, shrimp platter Marie-rose recorded odds of 1.24 with $95 \%$ confidence interval of $0.3394-4.5628$ while king prawns and lobsters platters recorded odds of 2.1 , with $95 \%$ confidence interval of 0.6190-7.1248. From the main course dishes, grass cutter and snail green soup recorded odds of 1.3 (Cl 0.3149-5.3222). Creamed potatoes, sunshine potatoes, stuffing and traditional accompaniments also had odds of 2.7 ( $\mathrm{Cl} \mathrm{0.6310-11.4881).}$ From the dessert category, sweets and profiteroles pyramid with respective odds of 2.7 (Cl 0.6310-11.4881) and 1.7 (Cl 0.5236-5.4384) showed apparent associations.

Three other food items did not appear to have associations. Mozzarella platters/salmon roll platters, crown of roast stuffed turkey with pan roasted juice, and cheese/ /biscuits showed no association with odds of 1.00 and $95 \%$ confidence intervals of 0.2603-3.8411, 0.3384-2.9548, and $0.3072-3.2554$, respectively.

The rest of the foods appeared protective with odds less than 1 (Table 4). They include: cream of tomato basil parmesan soup with croutons, assorted salads/suckling pig, cold cuts 
Table 4. Foods served at buffet with frequencies of illness, odds ratios (OR), and 95\% confidence intervals (Cl)

\begin{tabular}{llllll}
\hline Food eaten & No. who ate & No. ill & Per cent ill & P-value & OR (95\% CI) \\
\hline ST 1 & 17 & 10 & $58.82 \%$ & 0.3 & $0.61(0.2026-1.8922)$ \\
ST 2 & 11 & 5 & $45.45 \%$ & 0.7 & $1.24(0.3394-4.5628)$ \\
ST 3 & 14 & 5 & $35.71 \%$ & 0.2 & $2.10(0.6190-7.1248)$ \\
ST 4 & 10 & 5 & $50.00 \%$ & 1.0 & $1.00(0.2603-3.8411)$ \\
ST 5 & 8 & 5 & $62.50 \%$ & 0.4 & $0.56(0.1223-2.5636)$ \\
ST 6 & 9 & 5 & $55.56 \%$ & 0.7 & $0.77(0.1879-3.1753)$ \\
MC 1 & 18 & 9 & $50.00 \%$ & 1.0 & $1.00(0.3384-2.9548)$ \\
MC 2 & 29 & 17 & $58.62 \%$ & 0.2 & $0.53(0.2010-1.4394)$ \\
MC 3 & 9 & 4 & $44.44 \%$ & 0.7 & $1.29(0.3149-5.3222)$ \\
MC 4 & 10 & 3 & $30.00 \%$ & 0.1 & $2.69(0.6310-11.4881)$ \\
DS 1 & 16 & 11 & $68.75 \%$ & 0.08 & $0.36(0.1080-1.1805)$ \\
DS 2 & 10 & 3 & $30.00 \%$ & 0.1 & $2.69(0.6310-11.4881)$ \\
DS 3 & 15 & 6 & $40.00 \%$ & 0.3 & $1.69(0.5236-5.4384)$ \\
DS 4 & 10 & 6 & $60.00 \%$ & 0.4 & $0.62(0.1578-2.4413)$ \\
DS 5 & 14 & 7 & $50.00 \%$ & 1.0 & $1.00(0.3072-3.2554)$
\end{tabular}

of tenderloin of beef/cold turkey, grilled lobster and prawns with garlic butter slowly cooked a la plancha, assorted cheesecakes/Christmas cake, and fresh fruit platter. Again, confidence intervals include 1 and have wide margins conferring lack of significance and great variability on the observations.

\section{DISCUSSION}

Acute diarrhoea in adults is not rare; however, its incidence is not easy to determine because most cases are self-limiting and are often not reported. Where an acute diarrhoeal episode follows the ingestion of a meal or a drink and involves two or more people such as happened on the Sedco energy rig on the $25^{\text {th }}$ of December 2013, such an episode meets the Centres for Disease Control's (CDC) definition of a foodborne illness. With an incidence rate of 33 out of 190 people in 3 days, it also meets World Health Organisation's definition of an outbreak and thus requires an appropriate investigation.

Foodborne outbreaks can occur with any food type. However, in diarrhoeal outbreaks especially on ships, foods commonly implicated are sea foods [4]. Some of these foods were served in the buffet preceding this outbreak. Apparent associations seen with the shrimp, prawn and lobster dishes are therefore not surprising. What is surprising though is the apparent association of the outbreak with eating sweets. Sweets have not been shown to be implicated in diarrhoeal outbreaks before and this finding is a demonstration of the random nature of the outbreak and the possibility of a source outside the served foods. It is not surprising though that these associations lack statistical significance.
In the event of an exposure to disease transmitting agents, not all exposed individuals will develop the disease. Host factors and agent characteristics often play an important role in susceptibility and determining the success or otherwise of the transmission. Host factors such as age, sex, race, nutritional status, etc. may influence the susceptibility of the host in certain disease transmission mechanisms but in diarrhoeal outbreak situations, nutritional status may perhaps be the most important factor since it has bearings on immunity. Agent characteristics such as virulence and dose are also important determinants of the ability of the agent to cause a disease. In an outbreak situation such as this, successful transmission will depend on who came into contact with (ingested) the agent and the interplay between these agent factors and host factors. It is expected therefore that the pattern of distribution of cases in this outbreak will be uniform across all ages, races, and gender; depending on who ate which food.

Just as was anticipated, these variables did not seem to influence the distribution of the outbreak. Though there was a $100 \%$ male distribution, this was due to the fact that there were no females on board during the outbreak. The prevalence of Caucasians in the distribution of cases seems to suggest that Caucasians were more susceptible than other races. No literature however exists that describes a higher level of susceptibility among Caucasians in diarrhoeal outbreaks. In this study also, bivariate analysis with race as an exposure variable did not show any difference between Caucasians and other races (odds ratio 1.1; $\mathrm{Cl} 0.34-2.47$ ) 
even though frequency of cases is higher among Caucasians (Table 2). This observation is therefore just a random finding and can only be attributed to the fact that Caucasians have the highest population on the rig, forming about $90 \%$ of the rig's POB.

Symptom distribution in this outbreak was also just as expected. Gastroenteritis is characterised by diarrhoea with or without vomiting, and abdominal pain. Depending on the aetiologic agent, fever may be a sign as well. The nature of these symptoms and any other additional symptoms depend on the specific cause of the disease. There is no published general pattern of distribution of these symptoms in diarrhoeal outbreaks and therefore, the distribution of symptoms, as demonstrated by the present investigation, was most likely accidental and cannot lead to any conclusions.

While investigating and taking precautions to halt the outbreak, cases were mainly treated with oral rehydration salts and majority had their symptoms resolved in $24 \mathrm{~h}$. Very few required anti-motility agents to facilitate their return to work. This line of treatment was in keeping with standard management protocols for diarrhoea [5]. Dehydration, electrolyte depletion and acute renal failure are the main causes of death in cases of diarrhoea [6]. The most important treatment therefore is adequate and early rehydration. Antibiotics are rarely necessary and anti-motility agents are not recommended in most cases except when absolutely necessary.

The epidemic curve showed several peaks, but generally rose sharply to a peak and then assumed a downward trend. Each peak appears to be within a $12 \mathrm{~h}$ period; the incubation period of the common organisms responsible for diarrhoea outbreaks (bacteria and viruses). It points to a possible person to person transmission in a propagated fashion. The typical shape of a propagated epidemic curve where progressive peaks have increasing heights is however lost probably due to the early intervention to bring the outbreak under immediate control.

Three different food items, one from each group (starters, main course, and desserts) had odds ratios suggestive of associations with the outbreak. However, these associations turned out to be non-significant, as the p-values were all greater than 0.05 , and confidence intervals for each odds ratio included 1 (Table 4). Assuming these associations were significant, possible reasons why three different foods will be associated with the outbreak could be explained in two ways; either all three were contaminated, or this observation is because people ate different foods from different categories. At this level, no further analysis could have broken the tie and a food culture of the three implicating foods would have been the way to determine the particular food item responsible.
Most odds ratios in the study were also associated with wide confidence intervals (Table 4). The wide range of plausible values for the odds ratios shown by these wide confidence intervals is a depicter of an extent of unreliability in the results. There are two possible reasons for an observation like this; either the data generally has an inherent variability or the sample size was too small [7]. In the case of this study, either of the two reasons could apply. The cause of the outbreak is probably not from any of the food items but from other sources such as contaminated door handles or guard rails. On the other hand, the sample size of 66 may be too small to give a more precise result and is an important limitation to the study. A solution may have been to increase the number of controls to increase the sample size. The fact that p-values show a general lack of significance in all the odds ratios point to a conclusion in the direction of the former reason; the outbreak was probably transmitted through sources remote from the foods served.

Door handles, wash room basins, hand rails, etc. have a high potential for contamination and can propagate diarrhoeal outbreaks $[1,8]$, especially in enclosed environments such as this one. The propagated picture of the epidemic curve in this outbreak coupled with the fact that the outbreak was rapidly brought under control with measures such as disinfection of surfaces, and hand washing makes this mode of transmission the most likely, and probably exonerates the foods served at the buffet.

The importance of hand washing and other basic hygiene practices in controlling diarrhoea and other communicable disease transmission was demonstrated during this outbreak. With hand washing and disinfection of handrails and door handles, the outbreak was brought under control. It is biologically plausible for transmission of diarrhoeal agents from person to person through these items and the practice of routine hand washing alone is enough to significantly reduce the risk of such infections [9].

\section{LIMITATIONS OF THE STUDY}

Inability to test food samples was a major setback to the success of the investigation. This would have helped identify any specific food items that may have been contaminated and would have added depth to the statistical. Added to this limitation, was the inability to collect more controls. Enrolling more controls would have increased the sample size and improved the reliability of the results. This could not be done because of the high change over rate of personnel on the rig. More than half of the people on board during the buffet left back to town within two days with new ones coming on board. More controls could therefore not be recruited. 


\section{CONCLUSIONS}

The data collected in this diarrhoea outbreak investigation showed three food types with odds ratios suggestive of an association with the outbreak. However, this lacked significance as $p$-values of the various odds ratios were high $(p>0.05)$ and confidence intervals showed low precision and included 1 in the range of possible values. These associations could therefore have happened by chance. The outbreak therefore had no association with the foods served at the buffet. The epidemic curve showed multiple peaks in a propagated fashion, each of which had durations of approximately $12 \mathrm{~h}$. This coincides with the incubation period of most microorganisms known to cause diarrhoeal outbreaks. Following intense public education on personal hygiene and the utilisation of techniques such as hand washing and sanitisation, disinfection of door handles and hand rails, etc. the outbreak rapidly subsided. The conclusion therefore is that this outbreak most probably resulted from person to person contact and faecal contamination of surfaces and door handles. The foods served at the buffet did not play a role in transmitting the disease.

Following from the results and conclusion, we recommend that regular public education campaigns emphasising personal hygiene and the need for regular hand washing should be carried out on rigs. We again recommend that housekeeping on rigs should include disinfection of surfaces and handles to prevent occurrence of diarrhoeal outbreaks.

\section{ACKNOWLEDGEMENTS}

The authors are grateful to the participants of the investigation.

\section{REFERENCES}

1. Zhang N, Miao R, Huang H, et al. Contact infection of infectious disease onboard a cruise ship. Sci Rep. 2016; 6: 38790, doi: 10.1038/ srep38790, indexed in Pubmed: 27929141.

2. Fujita DM, Henrique L, Giraldi RD, et al. Brazilian Public Health Policy for Cruise Ships - A Review of Morbidity and Mortality Rates - 2009/2015. Int J Travel Med Glob Health. 2018; 6(1): 11-15, doi: 10.15171/ijtmgh.2018.03.

3. Hameed JM, McCaffrey RL, McCoy A, et al. Incidence, Etiology and Risk Factors for Travelers' Diarrhea during a Hospital Ship-Based Military Humanitarian Mission: Continuing Promise 2011. PLoS One. 2016; 11(5): e0154830, doi: 10.1371/journal.pone.0154830, indexed in Pubmed:27171433.

4. Rooney RM, Cramer EH, Mantha S, et al. A review of outbreaks of foodborne disease associated with passenger ships: evidence for risk management. Public Health Rep. 2004; 119(4): 427-434, doi: 10.1016/j.phr.2004.05.007, indexed in Pubmed: 15219800.

5. Republic of Ghana. Standard Treatment Guidelines. Ministry of Health. Sixth Edition 2010.

6. World Health Organization. WHO Diarrhoeal disease Fact sheet. WHO Media Centre, 2017. http://www.who.int/mediacentre/ factsheets/fs330/en/.

7. Cumming G, Finch S. Inference by eye: confidence intervals and how to read pictures of data. Am Psychol. 2005; 60(2): 170-180, doi: 10.1037/0003-066X.60.2.170, indexed in Pubmed: 15740449.

8. Carling PC, Bruno-Murtha LA, Griffiths JK. Cruise ship environmental hygiene and the risk of norovirus infection outbreaks: an objective assessment of 56 vessels over 3 years. Clin Infect Dis. 2009; 49(9): 1312-1317, doi: 10.1086/606058, indexed in Pubmed: 19814610.

9. Curtis V, Cairncross S. Effect of washing hands with soap on diarrhoea risk in the community: a systematic review. Lancet Infect Dis. 2003; 3(5): 275-281, doi: 10.1016/S1473-3099(03)00606-6, indexed in Pubmed: 12726975. 